\title{
ERCC1 Expression-Based Randomized Phase II Study of Gemcitabine/Cisplatin Versus Irinotecan/Cisplatin in Patients with Advanced Non-small Cell Lung Cancer
}

\author{
Ji-Youn Han, MD, PhD \\ Geon Kook Lee, MD, PhD \\ Kun Young Lim, MD \\ Young Ju Lee, MD, PhD \\ Byung Ho Nam, PhD \\ Jin Soo Lee, MD, PhD
}

Center for Lung Cancer,

Research Institute and Hospital,

National Cancer Center, Goyang, Korea

\begin{abstract}
Purpose
We evaluated the clinical utility of excision repair cross-complementation group 1 (ERCC1) expression as a predictive biomarker for platinum-based chemotherapy in advanced nonsmall cell lung cancer (NSCLC).
\end{abstract}

\section{Materials and Methods}

Eligible patients were randomly assigned to the GP (gemcitabine $1,250 \mathrm{mg} / \mathrm{m}^{2}$ on days 1 and 8 , and cisplatin $75 \mathrm{mg} / \mathrm{m}^{2}$ on day 1 every 3 weeks) or IP (irinotecan $65 \mathrm{mg} / \mathrm{m}^{2}$ and cisplatin $30 \mathrm{mg} / \mathrm{m}^{2}$ on days 1 and 8 every 3 weeks) arm. The primary goal of this study was to compare the response rate (RR) of the GP and IP arms according to the ERCC1 expression level.

\section{Results}

A total of 279 patients were randomly assigned to the GP $(n=139)$ and IP $(n=140)$ arms, among which $63 \%$ were ERCC1-positive and 268 patients were assessable for the RR. The GP and IP arms did not differ significantly with respect to the RR (29.8\% vs. $27.0 \%$, respectively; $p=0.082$ ), median progression-free survival (PFS; 4.5 months vs. 3.9 months, respectively; $p=0.117$ ), and overall survival (OS; 16.5 months vs. 16.7 months, respectively; $p=0.313$ ). When comparing the efficacy between the ERCC1-positive and ERCC1-negative groups, there was no significant difference in the RR (GP, $28.2 \%$ vs. $32.6 \%$, respectively, $p=0.509 ;$ IP, 30.2\% vs. 21.6\%, respectively, $p=0.536$ ), median PFS (GP, 4.6 months vs. 5.0 months, respectively, $p=0.506$; IP, 3.9 months vs. 3.7 months, respectively, $p=0.748$ ), or median OS (GP, 18.6 months vs. 11.9 months, respectively, $p=0.070$; IP, 17.5 months vs. 14.0 months, respectively, $p=0.821$ ).

\section{Conclusion}

Immunohistochemical analysis of the ERCC1 expression level did not differentiate the efficacy of platinum-based chemotherapy in advanced NSCLC.
Center for Lung Cancer, Research Institute and Hospital, National Cancer Center,

323 Ilsan-ro, Ilsandong-gu, Goyang 10408, Korea

Tel: $82-31-920-1154$

Fax: 82-31-920-2587

E-mail: jymama@ncc.re.kr

Received August 10, 2016

Accepted September 22, 2016

Published Online October 11, 2016
Key words

ERCC1, Platinum, Non-small-cell lung carcinoma

\section{Introduction}

Lung cancer remains the leading cause of cancer-related mortality worldwide. Non-small cell lung cancer (NSCLC) accounts for approximately $85 \%$ of all lung cancers, and $40 \%$ of these patients present at an advanced stage [1]. Recent advances in the understanding of the molecular origins of NSCLC have changed the treatment paradigm according to the target oncogenes [2]. However, the benefit of such target therapies remains limited to less than half of all patients with NSCLC; therefore, platinum-based chemotherapy has remained the mainstay in the treatment of NSCLC. Several large randomized studies have demonstrated the relative equivalence of platinum-based doublets, with response rates (RRs) ranging from $16 \%$ to $32 \%$ and a median survival of $8-11$ months, with 1- and 2-year survival rates of $35 \%$ to $45 \%$, and $10 \%$ to $20 \%$, respectively. However, most patients gen- 
erally experience disease progression after a median of 3-4 months of initiating chemotherapy, and the long-term prognosis remains poor $[3,4]$. Furthermore, no consensus has been reached for biomarkers predicting a benefit from platinum-based chemotherapy in advanced NSCLC [5].

Excision repair cross-complementation group 1 (ERCC1) plays an essential role in nucleotide excision repair, which removes platinum-DNA adducts [6]; therefore, high ERCC1 levels are usually associated with a lack of clinical benefit with platinum-based chemotherapy [7-10]. The association between ERCC1 expression and cisplatin resistance has been confirmed by ERCC1 immunohistochemistry analysis of postoperative tumor samples from the International Adjuvant Lung Cancer (IALT) trial. These analyses revealed that adjuvant chemotherapy significantly prolonged survival in patients with ERCC1-negative tumors relative to those with ERCC1-positive tumors [11]. Following publication of the ERCC1 protein expression level as a biomarker of survival benefit and treatment efficacy for cisplatin-based adjuvant chemotherapy, numerous studies tested the predictive or prognostic role of ERCC1 expression levels in advanced NSCLC. Some of these studies have suggested that ERCC1 expression levels are associated with a clinical benefit of platinum-based chemotherapy in advanced NSCLC [12,13]. However, these studies investigated patients treated with high ERCC1 levels using a non-platinum regimen, while none have compared the efficacy of platinum chemotherapy according to the ERCC1 level in advanced NSCLC. Thus, we prospectively investigated the role of ERCC1 levels for predicting the efficacy of two different platinum-based regimens.

To date, most platinum-based regimens have demonstrated similar efficacy in unselected patients with advanced NSCLC [3]. Moreover, gemcitabine and cisplatin (GP) and irinotecan and cisplatin (IP) regimens showed similar efficacy in advanced NSCLC. [4] Although some studies have shown an inverse correlation between ERCC1 levels and efficacy toward GP, none have investigated ERCC1 level and the efficacy toward IP regimen in NSCLC. Interestingly, high ERCC1 levels were reportedly associated with irinotecan efficacy in advanced colorectal cancer [14]. Thus, we hypothesized that IP and GP regimens show different efficacy according to ERCC1 levels.

To date, immunohistochemical analysis using the mouse monoclonal antibody $8 \mathrm{~F} 1$ has been the most commonly used technique for measuring ERCC1 protein expression. Given the feasibility of immunohistochemical analysis of ERCC1 in biopsy samples, we investigated the clinical usefulness of ERCC1 as a predictive biomarker for two different platinumbased regimens in advanced NSCLC.

\section{Materials and Methods}

\section{Eligibility criteria}

The main eligibility criteria included histological confirmation of advanced NSCLC, no prior chemotherapy, age $\geq 18$ years, an Eastern Cooperative Oncology Group (ECOG) performance status (PS) $<2$, and measurable disease according to the Response Evaluation Criteria in Solid Tumors (RECIST). Additionally, adequate hematologic (white blood cell count $\geq 4,000 / \mathrm{mm}^{3}$, platelet count $\geq 150,000 / \mathrm{mm}^{3}$ ), hepatic (bilirubin level $\leq 1.5 \mathrm{mg} / \mathrm{dL}$, aspartate aminotransferase / alanine transaminase $\leq 80 \mathrm{IU} / \mathrm{L}$ ), and renal (creatinine concentration $\leq 1.5 \mathrm{mg} / \mathrm{dL}$ ) function was required. Patients with brain metastases were enrolled if they were clinically stable without steroid treatment. The exclusion criteria included serious concomitant systemic diseases and second primary malignancies within the preceding 5 years. The protocol was approved by an independent ethics committee/institutional review board, and the study was conducted in accordance with the Declaration of Helsinki and Good Clinical Practice. Each patient provided written informed consent (NCT01003964).

\section{Study design}

This was an open-label, randomized phase II trial that compared the efficacy of GP versus IP chemotherapy in chemo-naïve advanced NSCLC patients according to ERCC1 expression level. To minimize the impact of subsequent therapy on overall survival (OS), second- or third-line therapies were predefined. Patients with non-squamous cell lung cancer randomly received pemetrexed followed by docetaxel or docetaxel followed by pemetrexed as the second- or thirdline treatment when the disease progressed. Patients with squamous cell lung cancer received docetaxel as a secondline therapy.

\section{Random assignment and treatment plan}

After ERCC1 assessment, patients were randomly assigned to either the IP or GP arm using the random blocksize permutation method based on a computer-generated block randomization schedule. Patients in the IP arm received $65 \mathrm{mg} / \mathrm{m}^{2}$ irinotecan and $30 \mathrm{mg} / \mathrm{m}^{2}$ cisplatin on days 1 and 8 every 3 weeks. Patients in the GP arm received $1,250 \mathrm{mg} / \mathrm{m}^{2}$ gemcitabine on days 1 and 8 and $75 \mathrm{mg} / \mathrm{m}^{2}$ cisplatin on day 1 every 3 weeks. Patients were treated for a maximum of nine cycles or until reaching progressive disease (PD), death, or unacceptable toxicity.

As the disease progressed, patients with non-squamous 
cell lung cancer were randomly assigned to receive either pemetrexed (500 $\mathrm{mg} / \mathrm{m}^{2}$ every 3 weeks) or docetaxel (75 $\mathrm{mg} / \mathrm{m}^{2}$ every 3 weeks). Patients with squamous cell lung cancer received docetaxel as second-line therapy with disease progression. The randomization process is shown in S1 Fig.

\section{Study assessment}

The safety assessment included patient history, physical examination, vital signs, ECOG PS, adverse effects and electrocardiography blood chemistry and hematology findings. Safety assessments were performed upon screening, on day 1 of subsequent cycles, and during the final study visit using Common Terminology Criteria for Adverse Events (CTCAE) ver. 3.0 [15].

Objective tumor responses were assessed using RECIST ver. 1.0 [16] after every two cycles of therapy. Progressionfree survival (PFS) was calculated from the date of random assignment to PD or death. OS was calculated from the date of random assignment to death or the last follow-up.

\section{Immunohistochemistry for ERCC1 expression}

This study required the collection of formalin-fixed paraffin-embedded tumor blocks before therapy. The primary antibody for the detection of ERCC1 was clone 8F1 (catalog
No. GTX22356 from GeneTex, Irvine, CA). One pathologist (G.K.L.) who was unaware of the clinical data independently evaluated ERCC1 staining under a light microscope at a magnification of $\times 400$. Staining intensity was graded on a scale of 0 to 3 . Five images of representative areas were acquired at a magnification of $\times 400$ for each specimen, and a total of 500 to 1,500 positive or negative tumor nuclei per specimen were manually counted. The percentage of positive tumor nuclei was calculated for each specimen, and a proportion score was assigned ( 1 if $0 \%, 2$ if $0<$ to $\leq 10 \%, 3$ if 10 $<$ to $\leq 25 \%, 4$ if $25<$ to $\leq 50 \%$, and 5 if $>50 \%$ ). This proportion score was multiplied by the staining intensity of nuclei to obtain a final semiquantitative $\mathrm{H}$ score. Tumors with an $\mathrm{H}$ score exceeding 15 (i.e., tumors with a staining intensity score of 3 and $50 \%$ or more positive nuclei) were deemed ERCC1 positive.

\section{EGFR and KRAS mutations and anaplastic lymphoma kinase-fluorescence in situ hybridization analysis}

We analyzed EGFR and KRAS mutations using a polymerase chain reaction-based direct DNA sequencing method [17]. Anaplastic lymphoma kinase $(A L K)$ rearrangements were detected by fluorescent in situ hybridization in formalin-fixed paraffin-embedded specimens using the breakapart probe for the ALK gene (Vysis LSI ALK Dual Color, Abbott Molecular, Abbott Park, IL) [18].

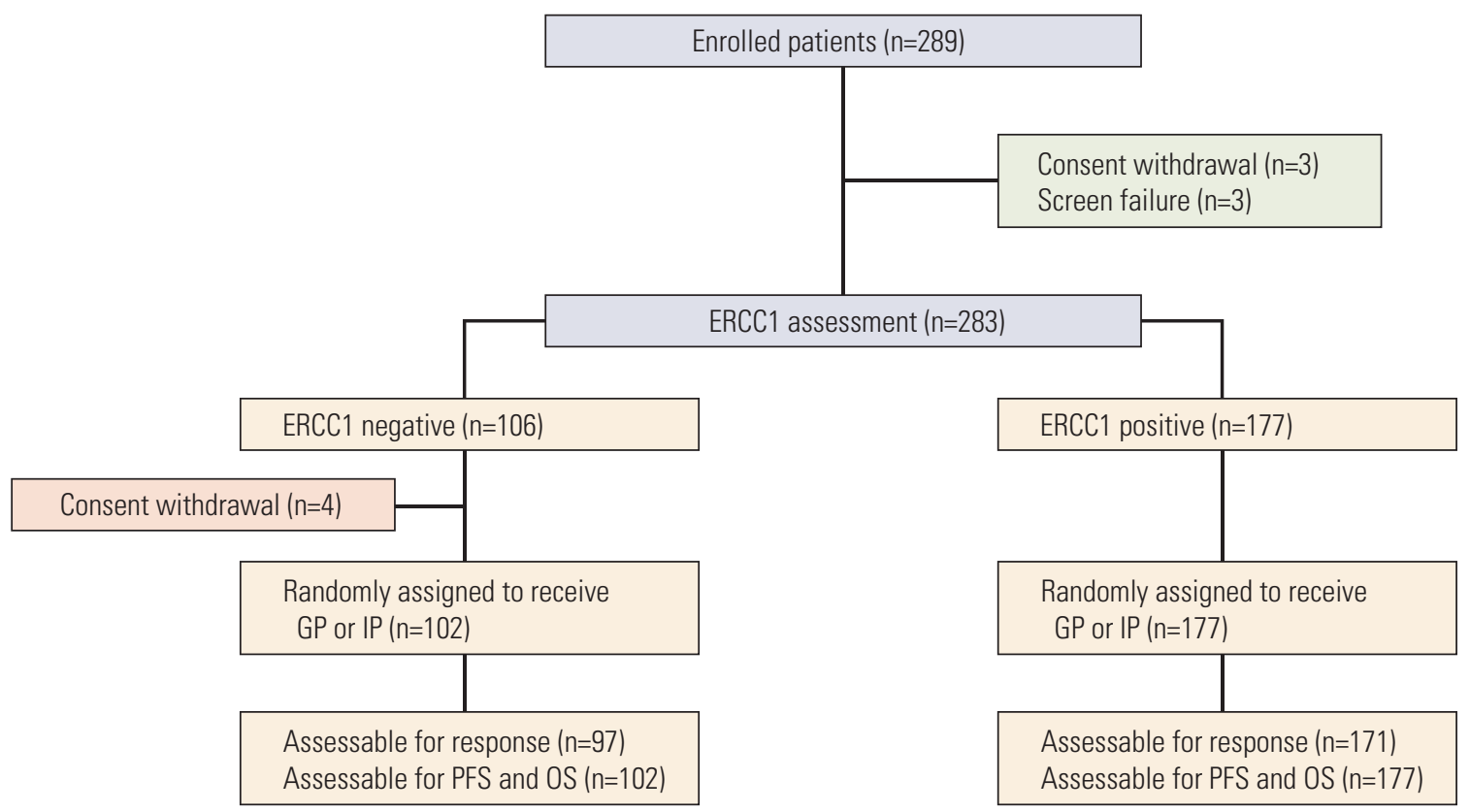

Fig. 1. CONSORT diagram. ERCC1, excision repair cross-complementation group 1; GP, gemcitabine and cisplatin; IP, irinotecan and cisplatin; PFS, progression-free survival; OS, overall survival. 
Table 1. Patient demographics and disease characteristics

\begin{tabular}{|c|c|c|c|c|}
\hline Variable & $\begin{array}{l}\text { All patients } \\
\quad(n=279)\end{array}$ & $\begin{array}{l}\text { GP arm } \\
(n=139)\end{array}$ & $\begin{array}{l}\text { IP arm } \\
(n=140)\end{array}$ & p-value \\
\hline \multicolumn{5}{|l|}{ Sex } \\
\hline Male & $181(64.9)$ & $91(65.5)$ & $90(64.3)$ & 0.900 \\
\hline Female & $98(35.1)$ & $48(34.5)$ & $50(35.7)$ & \\
\hline Age, median (range, yr) & $59(28-82)$ & $58(28-79)$ & $60(32-82)$ & 0.797 \\
\hline \multicolumn{5}{|l|}{ Smoking status } \\
\hline Current & $100(35.8)$ & $49(35.3)$ & $51(36.4)$ & 0.916 \\
\hline Former & $76(27.2)$ & $37(26.6)$ & $39(27.9)$ & \\
\hline Never & $103(36.9)$ & $53(38.1)$ & $50(35.7)$ & \\
\hline \multicolumn{5}{|l|}{ Histology } \\
\hline Adenocarcinoma & $215(77.1)$ & $109(78.4)$ & $106(75.7)$ & 0.664 \\
\hline Squamous cell & $45(16.1)$ & $22(15.8)$ & $23(16.4)$ & \\
\hline Large cell & $2(0.7)$ & 0 & $2(1.4)$ & \\
\hline Sarcomatoid & $3(1.1)$ & $1(0.7)$ & $2(1.4)$ & \\
\hline NOS & $14(5.0)$ & $7(5.0)$ & $7(5.0)$ & \\
\hline \multicolumn{5}{|l|}{ Stage } \\
\hline IIIB & $24(8.6)$ & $12(8.6)$ & $12(8.6)$ & 1.0 \\
\hline IV & $255(91.4)$ & $127(91.4)$ & $128(91.4)$ & \\
\hline \multicolumn{5}{|l|}{ ECOG PS } \\
\hline 0 & $21(7.5)$ & $15(10.8)$ & $6(4.3)$ & 0.102 \\
\hline 1 & $171(61.3)$ & $80(57.6)$ & $91(65.0)$ & \\
\hline 2 & $87(31.2)$ & $44(31.7)$ & $43(30.7)$ & \\
\hline \multicolumn{5}{|l|}{ ERCC1 } \\
\hline Negative & $102(36.6)$ & $49(35.3)$ & $53(37.9)$ & 0.710 \\
\hline Positive & $177(63.4)$ & $90(64.7)$ & $87(62.1)$ & \\
\hline \multicolumn{5}{|l|}{ EGFR mutations } \\
\hline 19DEL or L858R & $61(21.9)$ & $27(19.4)$ & $34(24.3)$ & 0.558 \\
\hline Rare exon 20 mutations & $6(2.2)$ & $3(2.2)$ & $3(2.1)$ & \\
\hline $\mathrm{T} 790 \mathrm{M}$ & $5(1.8)$ & $1(0.7)$ & $4(2.9)$ & \\
\hline Wild type & $150(53.8)$ & $78(56.1)$ & $72(51.4)$ & \\
\hline Not determined & $57(20.4)$ & $30(21.6)$ & $27(19.3)$ & \\
\hline \multicolumn{5}{|l|}{ KRAS mutations } \\
\hline G12X & $12(4.3)$ & $8(5.8)$ & $4(2.9)$ & 0.388 \\
\hline Wild type & $128(45.9)$ & $60(43.2)$ & $68(48.6)$ & \\
\hline Not determined & $139(49.8)$ & $71(51.1)$ & $68(48.6)$ & \\
\hline \multicolumn{5}{|l|}{ ALK-FISH } \\
\hline Positive & $4(1.4)$ & $2(1.4)$ & $2(1.4)$ & 0.819 \\
\hline Negative & $10(3.6)$ & $4(2.9)$ & $6(4.3)$ & \\
\hline Not determined & $265(95.0)$ & $133(95.7)$ & $132(94.3)$ & \\
\hline
\end{tabular}

GP, gemcitabine and cisplatin; IP, irinotecan and cisplatin; NOS, not otherwise specified; ECOG PS, Eastern Cooperative Oncology Group performance status; ERCC1, excision repair cross-complementation group 1; EGFR, epidermal growth factor receptor; ALK, anaplastic lymphoma kinase; FISH, fluorescent in situ hybridization.

\section{Statistical analysis}

The primary objective was to estimate the efficacy of GP or IP chemotherapy according to ERCC1 expression in patients with advanced NSCLC. The primary end point was the RR, which was defined as the proportion of patients whose best response was complete response or partial response among all per-protocol patients. The study employed a marker by treatment interaction design. We expected that the proportion of ERCC1-positive patients 
would be almost the same in both treatment groups. Moreover, it was expected that the ERCC1-negative group would show a better RR than the ERCC1-positive group. The expected RR for the ERCC-negative group was approximately $45 \%$, whereas a 30\% RR was expected for the ERCC1positive group. A total of 142 patients were needed for each group to detect a $15 \%$ difference in the RR between the ERCC1-positive and ERCC1-negative groups with 83\% power, a 5\% level of significance, and a one-sided test. The patients were randomly assigned to either the GP or IP arm at an equivalent ratio.

Additional end points included PFS, OS, and toxicities. All patients who received at least one cycle of chemotherapy were considered assessable for PFS, OS, and safety. All hypotheses were tested at a two-sided $\alpha$ level of 0.05 . The logrank test was used to compare PFS and OS according to the ERCC1 expression levels. The distribution of PFS and OS was estimated using the Kaplan-Meier method. Statistical comparison of the RRs according to mutation status was performed using chi-squared or Fisher exact tests. We also assessed interactions between treatment groups and ERCC1 expression subgroups in relation to RR and survivals, PFS and OS, using logistic regression and Cox regression test, respectively.

\section{Results}

\section{Patient and treatment characteristics}

Between February 2009 and September 2013, 289 patients were enrolled. Among 283 patients who underwent ERCC1 assessment, 177 patients $(62.5 \%)$ were ERCC1 positive, and 106 patients (37.5) were ERCC1 negative. Finally, 279 patients received treatment per protocol after ERCC1 assessment (Fig. 1). The characteristics of the 279 patients who were randomly assigned to each treatment arm are summarized in
Table 1. Most patients were male (64.9\%), ever smokers $(63.1 \%)$, and exhibited good PS $(68.8 \%)$, stage IV disease $(91.4 \%)$, and adenocarcinoma histology $(78.1 \%)$.

EGFR or KRAS mutation testing was not routinely performed at our institution at the time of the study initiation; thus, we retrospectively collected mutation data. The EGFR and KRAS mutation status were available in $222(79.6 \%)$ and $140(50.2 \%)$ patients, respectively, while there was no significant difference in the frequencies between treatment arms. We also did not find any significant association between EGFR or KRAS mutation status and ERCC1 level.

The median number of treatment cycles given to patients in both arms did not differ significantly relative to treatment assignment or ERCC1 expression (Mann-Whitney test; $\mathrm{p}=0.418$ and $\mathrm{p}=0.503$, respectively) (Table 2 ).

\section{Treatment outcome according to ERCC1 level and treat- ment arm}

Of the 279 patients in this study, 268 were assessable for a response. First, we compared the RR according to the treatment arms. The RR was $29.8 \%$ (95\% confidence interval [CI], 22.0 to 38.4) for the GP arm and 27.0\% (95\% CI, 19.8 to 35.3) for the IP arm ( $\mathrm{p}=0.082)$. When the RR was compared according to the ERCC1 level in each treatment arm, there were no significant differences (Table 3). We also compared the efficacy according to the ERCC1 level and found no significant differences. The RR was 29.2\% (95\% CI, 22.6 to 36.7) for the ERCC1-positive group and 26.8\% (95\% CI, 18.3 to 36.8) for the ERCC1-negative group ( $\mathrm{p}=0.741$ ). When the $R R$ was compared according to treatment arm in each ERCC1 group, there was no significant difference between the GP and IP arms in the ERCC1-positive group (28.2\% for GP vs. $30.2 \%$ for IP; $\mathrm{p}=0.362$ ). Additionally, the GP arm showed a trend toward a higher RR than the IP arm in the ERCC1-negative group ( $32.6 \%$ vs. $21.6 \%$, respectively; $\mathrm{p}=0.085$ ).

The cutoff for the OS update was June 29, 2015, and the median duration of follow-up was 16.7 months (range, 0.5 to 70.9 months). Of the 279 patients in this study, 266 patients

Table 2. Cycles of treatment by arm and assignment

\begin{tabular}{|c|c|c|c|c|}
\hline & \multicolumn{2}{|c|}{ GP } & \multicolumn{2}{|c|}{ IP } \\
\hline & ERCC1 positive & ERCC1 negative & ERCC1 positive & ERCC1 negative \\
\hline Mean & 4.2 & 3.9 & 3.8 & 3.5 \\
\hline Standard deviation & 2.2 & 1.8 & 2.2 & 1.6 \\
\hline Median & 4 & 4 & 4 & 4 \\
\hline Range & $1-9$ & $1-9$ & 1.9 & 1.9 \\
\hline
\end{tabular}

GP, gemcitabine and cisplatin; IP, irinotecan and cisplatin; ERCC1, excision repair cross-complementation group 1. 


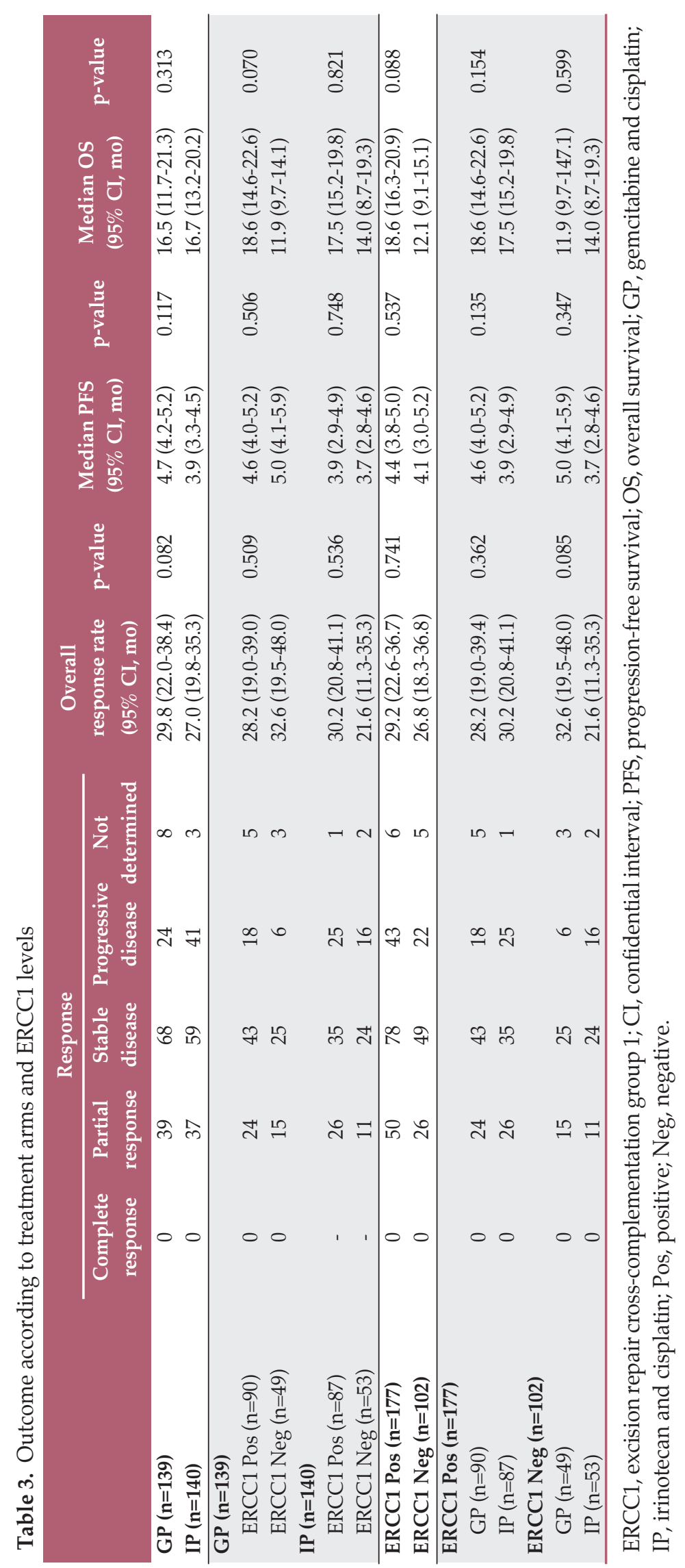

VOLUME 49 NUMBER 3 JULLY 2017 
Table 4. Subsequent treatment

\begin{tabular}{|c|c|c|c|}
\hline & GP $(n=139)$ & IP $(n=140)$ & p-value \\
\hline \multicolumn{4}{|l|}{ Second-line therapy } \\
\hline Pemetrexed & $40(28.8)$ & $39(27.9)$ & 0.189 \\
\hline Docetaxel & $35(25.2)$ & $51(36.4)$ & \\
\hline EGFR-TKI & $34(24.5)$ & $33(23.6)$ & \\
\hline Platinum-based doublet & $2(1.4)$ & $3(2.1)$ & \\
\hline Crizotinib & $2(1.4)$ & $1(0.7)$ & \\
\hline No treatment & $26(18.7)$ & $13(9.3)$ & \\
\hline \multicolumn{4}{|l|}{ Third-line therapy } \\
\hline Pemetrexed & $38(27.3)$ & $46(32.9)$ & 0.428 \\
\hline Docetaxel & $15(10.8)$ & $16(11.4)$ & \\
\hline EGFR-TKI & $31(22.3)$ & $26(18.6)$ & \\
\hline Doublet chemotherapy & $8(5.8)$ & $14(10.0)$ & \\
\hline ALK-TKI & $2(1.4)$ & $1(0.7)$ & \\
\hline Radiotherapy & $2(1.4)$ & $3(2.1)$ & \\
\hline Anti-PD1 therapy & 0 & $2(1.4)$ & \\
\hline No treatment & $43(30.9)$ & $32(22.9)$ & \\
\hline
\end{tabular}

Values are presented as number (\%). GP, gemcitabine and cisplatin; IP, irinotecan and cisplatin; EGFR, epidermal growth factor receptor; TKI, tyrosine kinase inhibitor; ALK, anaplastic lymphoma kinase.

Table 5. Adverse events $\geq$ grade 3

\begin{tabular}{lccc} 
Adverse event & IP & GP & p-value \\
Anemia & $14(9.8)$ & $22(15.7)$ & 0.155 \\
Leukopenia & $14(9.8)$ & $9(6.4)$ & 0.385 \\
Neutropenia & $58(40.6)$ & $53(37.9)$ & 0.715 \\
Thrombocytopenia & $2(1.4)$ & $12(8.6)$ & 0.006 \\
Diarrhea & $11(7.7)$ & 0 & 0.001 \\
Fatigue & $4(2.8)$ & $6(4.3)$ & 0.538 \\
Vomiting & $4(2.8)$ & $1(0.7)$ & 0.371 \\
\hline
\end{tabular}

IP, irinotecan and cisplatin; GP, gemcitabine and cisplatin.

(95.3\%) suffered disease progression or died. The median PFS and OS for all patients were 4.3 months (95\% CI, 3.7 to $4.9)$ and 16.7 months (95\% CI, 14.0 to 19.4$)$, respectively. When the PFS and OS were compared according to the ERCC1 level or treatment arm, there was no significant difference (Table 3, S2 Fig. A-D). Moreover, subgroup analysis revealed no significant differences between the GP and IP arms in the ERCC-positive (S3 Fig. A and B) or -negative group (S3 Fig. C and D). When the PFS were compared according to the ERCC1 level in the GP arm, no difference was observed (S4 Fig. A). However, the ERCC1-positive group showed a trend toward longer OS than the ERCC1negative group in the GP arm (18.6 months vs. 11.9 months, respectively; $\mathrm{p}=0.07$ ) (S4 Fig. B). In the IP arm, there was no significant difference in survival according to ERCC1 level (S4 Fig. C and D).

Interaction tests did not show a significant difference in RR, PFS, and OS between the ERCC1-positive and ERCC1negative group. The $p$-values for the interaction treatment with ERCC1 subgroup for RR, PFS, and OS were $0.248,0.233$, and 0.773 , respectively.

\section{Subsequent treatment}

Of the 279 patients in this study, $240(86 \%)$ and $204(73 \%)$ received second-line and third-line therapy, respectively. Of the 240 patients who received second-line therapy, 165 (69\%) received predefined second-line therapy. Of the 204 patients 
who received third-line therapy, 105 (51\%) received predefined third-line therapy. There was no significant difference in the subsequent treatments given to each arm (Table 4).

\section{Adverse events}

Anemia and neutropenia were the most commonly reported treatment-related adverse events. Overall, the grade 3 or 4 toxicity rates were low in both arms (Table 5). Grade 3 diarrhea was more common in the IP arm $(\mathrm{p}=0.001)$, while grade 3 or 4 thrombocytopenia was more common in the GP $\operatorname{arm}(\mathrm{p}=0.006)$. There were no treatment-related deaths.

\section{Discussion}

Treatment of advanced NSCLC has changed significantly since demonstration of the superior efficacy of target therapy against driver oncogenes such as EGFR mutations and ALK rearrangements. Nevertheless, platinum-based chemotherapy remains the standard therapy for advanced NSCLC without known driver mutations. At the time of this study initiation, platinum-based chemotherapy was the standard for advanced NSCLC in Korea. Routine EGFR mutation testing for selected first-line therapy started in April 2011 in Korea. In this study, we prospectively investigated the predictive value of ERCC1 expression level in advanced NSCLC patients who participated in a randomized phase II study comparing the efficacy of GP versus IP. Furthermore, our study predefined the subsequent treatment course to minimize the impact on OS. This design allowed us to test the prognosis and predictive value of the ERCC1 level in advanced NSCLC. However, we did not find any significant difference in RR, PFS, or OS according to the ERCC1 level in patients with advanced NSCLC.

Correlative biomarker analysis from two randomized phase III trials demonstrated that low ERCC1 protein or mRNA levels are associated with a better RR to platinumbased chemotherapy in advanced NSCLC. Nevertheless, both studies failed to demonstrate any significant association with survival $[12,13]$. Recently, another randomized phase III study investigated whether ERCC1 protein level-based chemotherapy selection would improve survival in advanced NSCLC; however, this investigation failed to demonstrate any differential benefit in RR or survival [19]. To date, patients with high ERCC1 levels have been assigned to non-platinum chemotherapy in ERCC1 level-based clinical trials for advanced NSCLC. However, no studies have compared the efficacy of the same platinum chemotherapy according to the ERCC1 level. Because the patients in our study received homogeneous platinum chemotherapy, we could prospectively investigate the predictive value of the ERCC1 level on platinum chemotherapy. However, we did not detect any correlation between the ERCC1 level and efficacy of GP or IP in advanced NSCLC. Moreover, the ERCC1 level was not prognostic in these patients.

Recently, a validation study using the ERCC1 $8 \mathrm{~F} 1$ antibody from two independent randomized trials of postoperative adjuvant cisplatin-based chemotherapy failed to validate ERCC1 protein expression as a predictive biomarker in NSCLC $[20,21]$. The authors suggested that a change in the performance of ERCC1 8 F1 antibody since 2006 resulted in discordance in ERCC1 staining, even in sample samples. Furthermore, they found that the currently available ERCC1 antibodies could not distinguish functional from non-functional isoforms, which may result in misclassification of tumors. Moreover, Schneider et al. [22] reported that the results of commercial ERCC1 testing are inconsistent and unreliable. These authors evaluated ERCC1 testing offered by three large commercial laboratories and found a significant difference in the independent laboratory ERCC1 expression rates $(70 \%$ vs. $60 \%$ vs. $44 \%$, p < 0.0001$)$. Furthermore, none of the assays could predict platinum resistance with a specificity greater than $50 \%$ [22]. Taken together, these findings suggest that ERCC1 testing is not applicable for routine practice in patients with NSCLC.

Because NSCLC patients are usually treated with combination chemotherapy, it may be necessary to assess multiple biomarkers. In addition to ERCC1, RRMI and BRAC1 levels have been investigated; however, none of these markers have demonstrated an adequate level of evidence for routine clinical use [23,24]. In addition to the ERCC1 expression levels, ERCC1 polymorphisms have been evaluated for cisplatin sensitivity in NSCLC. A meta-analysis of 1,252 NSCLC patients reported that there was no significant link between ERCC1 polymorphism and cisplatin sensitivity [25].

Despite the considerable potential for use of ERCC1 as a biomarker for cisplatin sensitivity, our study showed that immunohistochemical staining of ERCC1 is not adequate for selecting patients for platinum-based chemotherapy in advanced NSCLC. Further efforts are needed to develop clinically useful biomarkers to determine sensitivity to platinumbased chemotherapy in NSCLC.

\section{Conclusion}

There was no significant difference in efficacy between GP and IP treatment according to the ERCC1 expression level of the patient. Immunohistochemical analysis of the ERCC1 
expression level did not differentiate the efficacy of platinum-based chemotherapy in advanced NSCLC.

\section{Conflicts of Interest}

Conflict of interest relevant to this article was not reported.

\section{Electronic Supplementary Material}

Supplementary materials are available at Cancer Research and Treatment website (http://www.e-crt.org).

\section{Acknowledgments}

This study was supported by grants 1410660 and 1410540 from the National Cancer Center.

\section{References}

1. Cufer T, Ovcaricek T, O'Brien ME. Systemic therapy of advanced non-small cell lung cancer: major-developments of the last 5-years. Eur J Cancer. 2013;49:1216-25.

2. Cooper WA, O'Toole S, Boyer M, Horvath L, Mahar A. What's new in non-small cell lung cancer for pathologists: the importance of accurate subtyping, EGFR mutations and ALK rearrangements. Pathology. 2011;43:103-15.

3. Schiller JH, Harrington D, Belani CP, Langer C, Sandler A, Krook J, et al. Comparison of four chemotherapy regimens for advanced non-small-cell lung cancer. N Engl J Med. 2002;346: 92-8.

4. Ohe Y, Ohashi Y, Kubota K, Tamura T, Nakagawa K, Negoro $\mathrm{S}$, et al. Randomized phase III study of cisplatin plus irinotecan versus carboplatin plus paclitaxel, cisplatin plus gemcitabine, and cisplatin plus vinorelbine for advanced non-small-cell lung cancer: Four-Arm Cooperative Study in Japan. Ann Oncol. 2007;18:317-23.

5. Rose MC, Kostyanovskaya E, Huang RS. Pharmacogenomics of cisplatin sensitivity in non-small cell lung cancer. Genomics Proteomics Bioinformatics. 2014;12:198-209.

6. Bergstralh DT, Sekelsky J. Interstrand crosslink repair: can XPF-ERCC1 be let off the hook? Trends Genet. 2008;24:70-6.

7. Zheng Z, Chen T, Li X, Haura E, Sharma A, Bepler G. DNA synthesis and repair genes RRM1 and ERCC1 in lung cancer. N Engl J Med. 2007;356:800-8.

8. Lord RV, Brabender J, Gandara D, Alberola V, Camps C, Domine $\mathrm{M}$, et al. Low ERCC1 expression correlates with prolonged survival after cisplatin plus gemcitabine chemotherapy in non-small cell lung cancer. Clin Cancer Res. 2002;8:2286-91.

9. Chen S, Zhang J, Wang R, Luo X, Chen H. The platinum-based treatments for advanced non-small cell lung cancer, is low/ negative ERCC1 expression better than high/positive ERCC1 expression? A meta-analysis. Lung Cancer. 2010;70:63-70.

10. Vilmar AC, Santoni-Rugiu E, Sorensen JB. ERCC1 and histopathology in advanced NSCLC patients randomized in a large multicenter phase III trial. Ann Oncol. 2010;21:1817-24.

11. Olaussen KA, Dunant A, Fouret P, Brambilla E, Andre F, Had- dad V, et al. DNA repair by ERCC1 in non-small-cell lung cancer and cisplatin-based adjuvant chemotherapy. N Engl J Med. 2006;355:983-91.

12. Cobo M, Isla D, Massuti B, Montes A, Sanchez JM, Provencio $\mathrm{M}$, et al. Customizing cisplatin based on quantitative excision repair cross-complementing 1 mRNA expression: a phase III trial in non-small-cell lung cancer. J Clin Oncol. 2007;25: 2747-54.

13. Reynolds C, Obasaju C, Schell MJ, Li X, Zheng Z, Boulware D, et al. Randomized phase III trial of gemcitabine-based chemotherapy with in situ RRM1 and ERCC1 protein levels for response prediction in non-small-cell lung cancer. J Clin Oncol. 2009;27:5808-15.

14. Vallbohmer D, Iqbal S, Yang DY, Rhodes KE, Zhang W, Gordon $\mathrm{M}$, et al. Molecular determinants of irinotecan efficacy. Int J Cancer. 2006;119:2435-42.

15. Trotti A, Colevas AD, Setser A, Rusch V, Jaques D, Budach V, et al. CTCAE v3.0: development of a comprehensive grading system for the adverse effects of cancer treatment. Semin Radiat Oncol. 2003;13:176-81.

16. Sohaib SA, Turner B, Hanson JA, Farquharson M, Oliver RT, Reznek RH. CT assessment of tumour response to treatment: comparison of linear, cross-sectional and volumetric measures of tumour size. Br J Radiol. 2000;73:1178-84.

17. Han JY, Kim SH, Lee YS, Lee SY, Hwang JA, Kim JY, et al. Comparison of targeted next-generation sequencing with conventional sequencing for predicting the responsiveness to epidermal growth factor receptor-tyrosine kinase inhibitor (EGFR-TKI) therapy in never-smokers with lung adenocarcinoma. Lung Cancer. 2014;85:161-7.

18. Kim H, Yoo SB, Choe JY, Paik JH, Xu X, Nitta H, et al. Detection of ALK gene rearrangement in non-small cell lung cancer: a comparison of fluorescence in situ hybridization and chromogenic in situ hybridization with correlation of ALK protein expression. J Thorac Oncol. 2011;6:1359-66.

19. Bepler G, Williams C, Schell MJ, Chen W, Zheng Z, Simon G, et al. Randomized international phase III trial of ERCC1 and 
RRM1 expression-based chemotherapy versus gemcitabine/ carboplatin in advanced non-small-cell lung cancer. J Clin Oncol. 2013;31:2404-12.

20. Friboulet L, Olaussen KA, Pignon JP, Shepherd FA, Tsao MS, Graziano S, et al. ERCC1 isoform expression and DNA repair in non-small-cell lung cancer. N Engl J Med. 2013;368:1101-10.

21. Wislez M, Barlesi F, Besse B, Mazieres J, Merle P, Cadranel J, et al. Customized adjuvant phase II trial in patients with nonsmall-cell lung cancer: IFCT-0801 TASTE. J Clin Oncol. 2014; 32:1256-61.

22. Schneider JG, Farhadfar N, Sivapiragasam A, Geller M, Islam $\mathrm{S}$, Selbs E. Commercial laboratory testing of excision repair cross-complementation group 1 expression in non-small cell lung cancer. Oncologist. 2014;19:459-65.
23. Tantraworasin A, Saeteng S, Lertprasertsuke N, Arayawudhikul N, Kasemsarn C, Patumanond J. The prognostic value of ERCC1 and RRM1 gene expression in completely resected non-small cell lung cancer: tumor recurrence and overall survival. Cancer Manag Res. 2013;5:327-36.

24. Tiseo M, Bordi P, Bortesi B, Boni L, Boni C, Baldini E, et al. ERCC1/BRCA1 expression and gene polymorphisms as prognostic and predictive factors in advanced NSCLC treated with or without cisplatin. Br J Cancer. 2013;108:1695-703.

25. Yu D, Shi J, Sun T, Du X, Liu L, Zhang X, et al. Pharmacogenetic role of ERCC1 genetic variants in treatment response of platinum-based chemotherapy among advanced non-small cell lung cancer patients. Tumour Biol. 2012;33:877-84. 